\title{
Coercivity and exchange bias of ferromagnetic/antiferromagnetic multilayers
}

\author{
G. Scholten, K. D. Usadel, and U. Nowak* \\ Theoretische Physik, Universität Duisburg-Essen, 47048 Duisburg, Germany
}

\begin{abstract}
For a model system consisting of a ferromagnetic layer exchange coupled to an antiferromagnetic layer with a compensated interface, detailed mean-field-type calculations are performed. Both the coercive field and the exchange bias field are calculated. For the coercive field, a rather broad enhancement around the Néel temperature $T_{N}$ of the antiferromagnetic layer is found irrespective of whether the antiferromagnetic layer is structurally disordered or not, while exchange bias is only found for disordered systems. We show that the observed enhancement of the coercivity around $T_{N}$ also found experimentally and the occurrence of exchange bias are of different origin.
\end{abstract}

PACS number(s): 75.70.Cn, 75.60.-d, 75.50.Lk

\section{INTRODUCTION}

For multilayers consisting of a ferromagnet (FM) in contact with an antiferromagnet (AFM), a shift of the hysteresis loop along the magnetic field axis can occur which is called exchange bias (EB). It is observed that this effect is accompanied by an enhancement of the coercivity $\left(H_{\mathrm{c}}\right)$. While there is a vast literature on EB (for a review, see Ref. 1), less attention has been paid to this enhancement of $H_{\mathrm{c}}$ even though the latter effect might turn out to be relevant for future applications. ${ }^{2}$

By now it is well established that the occurrence of an exchange bias field is the result of an interfacial interaction between the ferromagnet and the antiferromagnet. Two requirements have to be fulfilled in order for EB to occur: first, there must be a net magnetization in the interface layer of the AFM which is exchange coupled to the FM, and secondly, this magnetization must be stable during field reversal. For compensated interfaces, in particular, the most important question is how these requirements are established. Since the so-called spin-flop coupling has been shown not to be able to support EB by its own accord, ${ }^{3}$ attempts to explain EB in compensated interface structures now focus on disorderlike structural defects at the interface, ${ }^{4}$ magnetic dilution, ${ }^{5}$ or structures composed of grains of different orientations or sizes. $^{6,7}$

Malozemoff ${ }^{4}$ in his pioneering work argued that an ideal interface is unrealistic and roughness gives rise to local random fields and consequently to stable domains in the AFM layer carrying an irreversible net interface magnetization at low temperatures. Recently, in a series of papers we have extended these considerations introducing a microscopic model $^{5,8,9}$ in which disorder is introduced via magnetic dilution not only in the interface but also in the bulk of the AFM. According to these investigations, EB has its origin in an irreversible AF interface magnetization which arises from volume domains stabilized by defects in the bulk of the AF. These volume domains then influence the spin structure at the interface. Strong support is given to this picture by experiments in which nonmagnetic impurities are added in a systematic and controlled way to the AFM layer ${ }^{5,10-12}$ to form and influence domains. Note that we do not make any assumptions with respect to size, form, or stability of these domains in the AFM layer. Rather, they form during the cooling process and their properties are determined by the dilution of the AFM layer.

In a different approach, ${ }^{6}$ the AFM layer is modeled as consisting of individual grains with small or vanishing intergrain coupling. Here also metastable configurations are observed $^{7}$ carrying an irreversible AFM interface magnetization and thus leading to EB. Thus, although different forms of disorder may change the results in detail, they lead to the same overall picture as far as the basic understanding of EB is concerned: what matters is the possibility of establishing a stable domain structure in the AFM carrying some irreversible net interface magnetization.

Although many features observed in EB systems are explained quite successfully within this so-called domain state model, less attention has been given to the enhancement of the coercivity observed in EB systems. In the present paper, we report on results obtained with a mean-field-type calculation for an EB system consisting of a FM monolayer in contact with an AFM film having a large anisotropy like $\mathrm{CoO}$ or $\mathrm{FeF}_{2}$ so that the AFM layer can be modeled as an Ising system. The AFM layer is magnetically diluted and different degrees of dilution are considered. Both the bias field $H_{\mathrm{eb}}$ and the coercivity $H_{\mathrm{c}}$ are calculated.

The results obtained for the bias fields are in qualitative agreement with those obtained previously with Monte Carlo simulations. For the coercivity we find, in agreement with experiments, a significant maximum of $H_{\mathrm{c}}$ around the blocking temperature at which $H_{\mathrm{eb}}$ vanishes. This maximum is analyzed in detail as a function of temperature for an ideal AFM layer as well as for different degrees of dilution.

Most of the results are obtained under the simplifying assumption that the exchange interaction in the FM layer is very large. Under this assumption, reversal of the FM layer to a good approximation is by coherent rotation, making it possible to obtain a number of analytic results for the fields $H_{\mathrm{c}}$ and $H_{\mathrm{eb}}$ from which more insight into the mechanisms of $\mathrm{EB}$ and coercivity is gained. From these analytic results we conclude, in agreement with earlier findings, ${ }^{3}$ that the appearance of $\mathrm{EB}$ and the enhancement of the coercivity in EB systems are of different origin; both can be present and they are not correlated, in contrast to Ref. 13. 
The results are supplemented by a calculation in which a finite exchange interaction is assumed in the FM layer. In this case, the mean-field equations for the AFM layer have to be complimented by the corresponding equations for the FM spins. These equations are solved numerically for a certain set of parameters showing that a finite FM exchange does not change the results significantly at low temperatures.

\section{GENERAL THEORY}

The Hamiltonian $\mathcal{H}$ of the system is the sum of three terms, $\mathcal{H}=\mathcal{H}_{\mathrm{F}}+\mathcal{H}_{\mathrm{AF}}+\mathcal{H}_{\text {int }}$, with

$$
\mathcal{H}_{\mathrm{F}}=-J_{\mathrm{F}} \sum_{\langle i, j\rangle} \vec{S}_{i} \cdot \vec{S}_{j}-\sum_{i}\left(D S_{i x}^{2}+S_{i x} H\right)
$$

denoting the Hamiltonian of the FM layer,

$$
\mathcal{H}_{\mathrm{AF}}=J_{\mathrm{AF}} \sum_{\langle i, j\rangle} \epsilon_{i} \epsilon_{j} \sigma_{i} \sigma_{j}-\sum_{i} \epsilon_{i} \sigma_{i} H
$$

denoting the Hamilton operator of the AFM layer, and

$$
\mathcal{H}_{\text {int }}=-J_{\text {int }} \sum_{i \in(\text { int })} \epsilon_{i} S_{i x} \sigma_{i}
$$

denoting the interaction energy of both, where the label $i$ runs over all spins in the AFM interface layer and we use the same index to enumerate the adjacent spin in the FM layer. Three-dimensional unit vectors $\vec{S}_{i}$ and Ising variables $\sigma_{j}$ denote spins in the FM and AFM layer, respectively. The magnetic field $\vec{H}$ is applied along the $x$ direction which is parallel to the easy axis of the FM layer (anisotropy constant $D>0$ ), while the $z$ direction is normal to the layers. We consider an AFM layer with quenched disorder, $\epsilon_{i}=0,1$, with probability $p$ and $1-p$, respectively, also having its easy axis along $x$. Furthermore, we consider nearest-neighbor interactions on a simple cubic lattice with exchange constants $J_{\mathrm{F}}>0$ and $J_{\mathrm{AF}}>0$ for the FM and the AFM, respectively, while $J_{\text {int }}$ stands for the exchange constant between FM and AFM.

Providing the Curie temperature is very large as compared to all other relevant energies, reversal of the FM is to a good approximation by coherent rotation (see also Ref. 14) and a temperature dependence of the magnetization in the ferromagnet can be neglected if we restrict ourselves to relatively low temperatures. This means that the magnetization of the FM layer acts as an external parameter on the AFM layer similar to the magnetic field applied. Thus, within this approximation, the free energy appears to be a function of external field and magnetization direction of the FM layer. For an Ising-type AFM, it only depends on its $x$ component, $S_{x}$, and it is given by

$$
\mathcal{F}\left(S_{x}\right)=-N l D S_{x}^{2}-N l H S_{x}-k_{\mathrm{B}} T \operatorname{Tr} e^{-\beta\left(\mathcal{H}_{\mathrm{AF}}+\mathcal{H}_{\text {int }}\right)},
$$

where $l$ is the number of FM monolayers and $N$ the number of spins in a FM monolayer.

In this paper, we mainly concentrate on this approximation which enables us to derive a number of results analytically resulting in a deeper understanding of the complex behavior of these exchange coupled FM/AFM layers.
The free energy given in Eq. (4) is a function of $S_{x}$ and the external field $H$. It expresses the free energy of the AFM spin system keeping these parameters fixed. As a function of $S_{x}$, it may have different local minima for fixed external field $H$. These local minima give rise to (metastable) branches in hysteresis loops. Indeed, starting with a sufficiently large field applied in the $x$ direction and $S_{x}=1$, the effective field acting on the AFM interface layer is $B+J_{\text {int }} S_{x}>0$. Decreasing the external field, we follow the descending branch of the hysteresis loop. For small enough external field, the free energy for $S_{x}=1$ becomes metastable, i.e., it will get larger than the free energy for $S_{x}=-1$. The system stays in the metastable minimum during a further decrease of the external field until this minimum vanishes at a certain field $H_{-}$at which the ferromagnet switches from $S_{x}=1$ to $S_{x}=-1$. This procedure is very similar to the Stoner-Wohlfarth scenario for switching of a magnetic particle. The only difference is that in the present case the FM magnetization is coupled to an AFM layer which may or may not be in thermal equilibrium depending on its dilution or disorder, see below. Similar discussion holds for the ascending branch of the hysteresis loop. We now elucidate this scenario in more detail restricting ourselves first to an ideal (undiluted) AFM layer.

\section{COERCIVITY FOR COUPLED FM/AFM LAYERS WITHOUT DILUTION}

The first derivative of the free energy with respect to $S_{x}$ in the undiluted case $\left(\epsilon_{i}=1\right.$ for all $\left.i\right)$ can be expressed in terms of the interface magnetization of the AFM layer,

$$
\mathcal{F}^{\prime}\left(S_{x}\right)=-2 N l D S_{x}-N l H-J_{\text {int }} \sum_{i \in \text { int }}\left\langle\sigma_{i}\right\rangle
$$

and the second derivative of $\mathcal{F}$ is given by

$$
\mathcal{F}^{\prime \prime}\left(S_{x}\right)=-2 N l D-\beta J_{\text {int }}^{2}\left\langle\left(\sum_{i \in \text { int }}\left(\sigma_{i}-\left\langle\sigma_{i}\right\rangle\right)\right)^{2}\right\rangle,
$$

where $\left\langle\sigma_{i}\right\rangle$ denotes a thermal average. This second derivative is negative showing that $\mathcal{F}^{\prime}$ is decreasing monotonously. Thus in the interval $-1<S_{x}<1$ the first derivative of free energy has one zero or no zeros. In the first case, $\mathcal{F}$ has two local minima at the end points, $S_{x}=-1,1$ and one maximum in between, and in the second case only one global minimum at one of these end points, a situation similar to the simple Stoner-Wohlfarth scenario. Consequently, the fields $H_{-}$and $H_{+}$at which the magnetization of the FM switches can be obtained in the same way, i.e., from $\mathcal{F}^{\prime}=0$ at $S_{x}=1$ and $S_{x}=-1$, respectively, and we obtain

$$
\begin{aligned}
& H_{-}=-2 D-J_{\mathrm{int}} m_{\mathrm{int}}\left(H_{-}, S_{x}=1\right) / l, \\
& H_{+}=2 D-J_{\mathrm{int}} m_{\mathrm{int}}\left(H_{+}, S_{x}=-1\right) / l,
\end{aligned}
$$

with $m_{\text {int }}=(1 / N) \sum_{i \in \text { int }} \epsilon_{i}\left\langle\sigma_{i}\right\rangle$ being the AFM interface magnetization. Note that these equations are exact as far as the AFM layer is concerned but fluctuations in the FM layer are neglected. In Eqs. (7), the magnetizations of the AFM interface layer enter. These magnetizations have to be calculated with fixed field applied and for both $S_{x}=1$ and $S_{x}=-1$, respectively. 

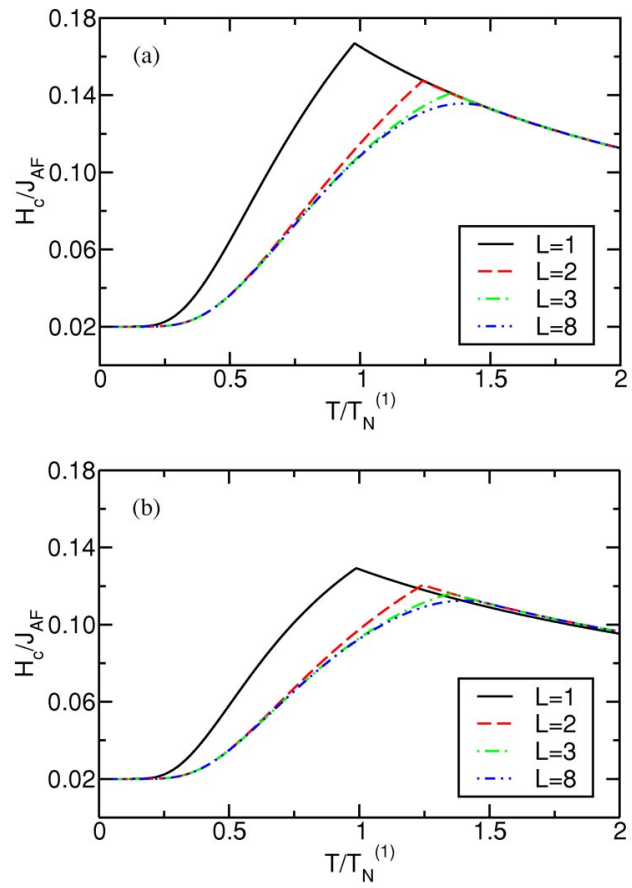

FIG. 1. (Color online) Coercive field as a function of reduced temperature for different AFM layer thicknesses $L$. (a) $J_{\text {int }}=-J_{\mathrm{AF}}$, (b) $J_{\text {int }}=J_{\mathrm{AF}}$.

For an ideal AFM layer, the induced magnetization in the AFM interface is completely reversible and it is an odd function of the effective field. Therefore, the coercive field is given by $H_{\mathrm{c}}=H_{+}=-H_{-}$and there is no exchange bias.

To obtain the induced interface magnetization of the AFM layer which influences the coercive field of the FM layer according to Eq. (7), we apply a mean-field approximation in the present paper. The mean-field equations for the local magnetization of the AFM spins, $\left\langle\sigma_{i}\right\rangle=m_{i}$, are given by

$$
m_{i}=\tanh \left[\beta\left(-J_{\mathrm{AF}} \sum_{j} m_{j}+J_{\mathrm{int}} S_{x}+H\right)\right] .
$$

Note that the second term in the brackets in Eq. (8) is present only if $i$ labels a spin of the AFM interface layer. For the other AFM spins, this term has to be omitted.

These mean-field equations have been solved numerically together with Eqs. (7) for obtaining the coercive field $H_{c}=H_{+}$. Results are shown in Fig. 1 for different AFM layer thicknesses and for positive and negative interlayer exchange interaction, respectively, with $D / J_{\mathrm{AF}}=0.01$. $L$ denotes the number of AFM monolayers and $T_{N}^{(1)}$ the Néel temperature of an ideal AFM monolayer.

For very low temperatures, the AFM layer is completely ordered with vanishing net magnetization at the interface so that the coercive field approaches the free FM layer value $2 D$. For increasing temperatures it is seen from Fig. 1 that due to the coupling of the AFM layer to the FM layer, a strong enhancement of the coercive field results. Its maximum is at or very close to the Néel temperature at which the coercive field has a cusp. Note that this enhancement is also present above the Néel temperature and that it decreases with increasing temperature only rather slowly. The enhancement of the coercive field decreases strongly below the Néel temperature when going from one to two AFM monolayers but it decreases only slowly when increasing the AFM thickness further. We expect that this behavior may change when considering an AFM layer with smaller anisotropy.

Within the present approximation in which the FM layer is described as a macrospin rotating coherently, the coercive field of the FM layer is independent of temperature and given by $2 D$ for vanishing coupling to the AFM layer. As can be seen from Fig. 1, the coupling to the AFM layer results in a remarkable enhancement of this field which is larger for antiferromagnetic interface coupling as compared to ferromagnetic coupling. With increasing AFM layer thickness, the Néel temperature shifts to higher temperatures-as expected-and the enhancement of the coercive field is reduced in agreement with experimental findings. ${ }^{15}$

If the coupling of the AFM layer to the FM layer is weak, the AFM interface magnetization can be linearized. Under this condition, explicit expressions for the coercive fields can be obtained from which a deeper insight into the complex behavior of these coupled systems can be gained.

The linearized induced interface magnetization contains two parts, one which is proportional to the sum of the external field and the exchange field from the FM layer and one which is proportional to the external field only. This second term arises indirectly from an exchange coupling of the AFM interface layer to its neighboring AFM monolayer which only sees the applied field resulting in a term linear in the external field. However, its contribution to the interface magnetization is very small (and absent for an AFM monolayer) and will be shown to be negligible for moderate values of $J_{\text {int }}$ resulting in the following approximate expression for $m_{\text {int }}$ in the linear regime:

$$
m_{\mathrm{int}}=\chi_{\mathrm{AF}}^{(1)}\left(H+J_{\mathrm{int}} S_{x}\right) .
$$

Thus, the AFM interface magnetization as a function of $H$ contains two branches during a hysteresis cycle. In the case of $J_{\text {int }}>0$, there is an upper branch for $S_{x}=1$ when reducing the external field and a lower branch after switching of the FM layer from $S_{x}=1$ to $S_{x}=-1$ when increasing the field again. For $J_{\text {int }}<0$, the behavior is reversed.

Within this linear approximation, an explicit equation for the coercive field $H_{c}$ can be obtained from Eqs. (7) and (9),

$$
H_{\mathrm{c}}=\frac{2 D+J_{\mathrm{int}}^{2} \chi_{\mathrm{AF}}^{(1)} / l}{1+J_{\mathrm{int}} \chi_{\mathrm{AF}}^{(1)} / l}
$$

where the susceptibility entering this equation is obtained from Eq. (8) with the following result for a single AFM monolayer:

$$
\chi_{\mathrm{AF}}^{(1)}=\frac{\beta}{\cosh ^{2}\left(\beta J_{\mathrm{AF}} z m_{s}^{(1)}\right)+\beta J_{\mathrm{AF}} z} .
$$

In the general case and neglecting the influence of the homogeneous magnetization of the neighboring AFM layer on the AFM interface layer this susceptibility is given by 


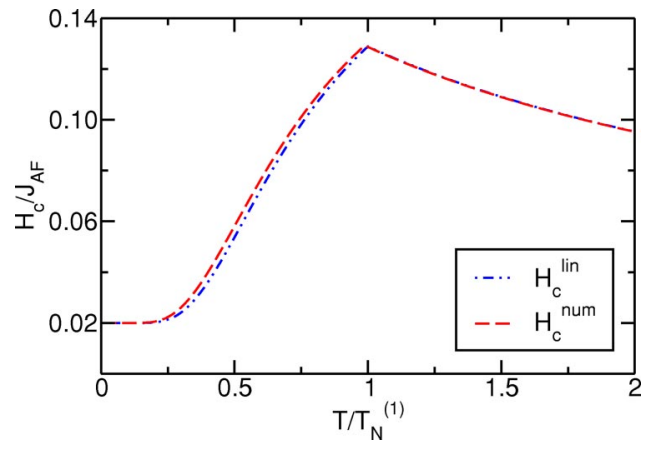

FIG. 2. (Color online) Comparison of the numerical solution and the linear approximation for AFM layer thicknesses $L=1$ as a function of temperature. $J_{\mathrm{int}}=J_{\mathrm{AF}}$.

$$
\chi_{\mathrm{AF}}^{(1)}=\frac{\beta}{\cosh ^{2}\left[\beta J_{\mathrm{AF}}\left(z m_{s}^{(1)}+m_{s}^{(2)}\right)\right]+\beta J_{\mathrm{AF}} z} .
$$

In these equations, the staggered magnetizations for $H=0$, $m_{s}^{(l)}$, enter where $l$ labels the AFM layers $(l=1$ being the AFM interface layer). These quantities have to be obtained numerically from the self consistency equations.

Equation (10) nicely illustrates that the coercive field depends on two contributions, one coming from the FM itself (2D) and one from the interaction with the AFM. The denominator depends on the sign of the interface coupling. This explains the fact that in Fig. $1, H_{c}$ is larger for negative interface coupling.

In Fig. 2, we show the coercive field as a function of temperature for an AFM monolayer obtained from the full numerical solution of the self-consistency equations and results obtained within the linear approximation. Both approaches give nearly identical results for $J_{\text {int }}=J_{\mathrm{AF}}$ and the same is true for smaller values of the interface coupling. However, with increasing strength of the interface coupling, the linear approximation gets worse, as can be seen from Fig. 3 , in which the coercive field is shown as a function of the interface coupling at that temperature at which the deviation of the linear approximation from the full numerical solution for $J_{\text {int }}=J_{\mathrm{AF}}$ is largest.

It is possible to generalize the present approach to systems in which the FM has not an infinite exchange interac-

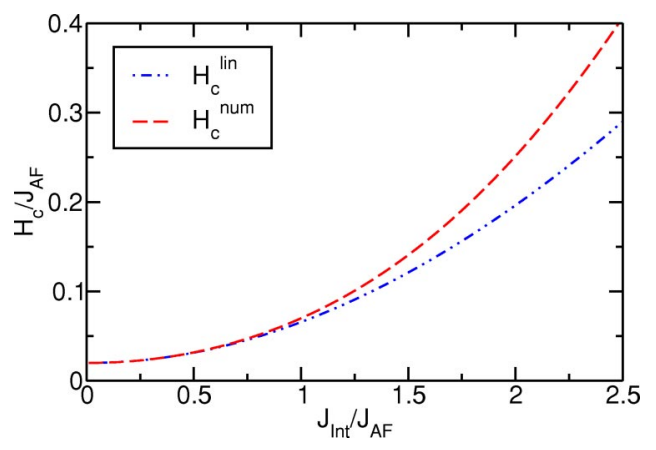

FIG. 3. (Color online) Comparison of the numerical solution and the linear approximation for AFM layer thicknesses $L=1$ as a function of reduced interface coupling at $T=0.56 T_{N}{ }^{(1)}$.

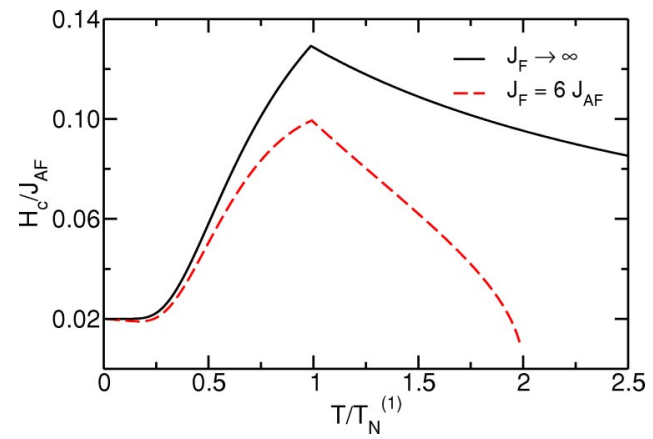

FIG. 4. (Color online) Coercive field as a function of reduced temperature for different exchange parameters of the FM. $L=1$, $J_{\text {int }}=J_{\mathrm{AF}}$.

tion, as has been assumed up to now, but a finite one. In this case we have to write down, in addition to the mean-field equations for the AFM, mean-field equations for the spins in the FM layer which we solved numerically for $L=1$. Results are shown in Fig. 4. The dashed line corresponds to a FM layer with finite exchange interaction while the solid line corresponds to a FM layer with very large $J_{F}$. For finite $J_{F}$, the magnetic order in the AFM layer decreases with increasing temperature and vanishes at the Curie temperature, $T_{c}=2 T_{N}$ in the present case. The coercive field of the uncoupled FM layer is expected to go smoothly from its zerotemperature value $0.02 J_{\mathrm{AF}}$ to zero at $T_{c}$. The remarkable enhancement of the coercive field observed in Fig. 4 again is due to the coupling to the AFM layer and it persists even for temperatures far above the Néel temperature, in agreement with experimental findings. ${ }^{16}$

\section{DILUTED SYSTEMS}

In previous work, we have shown with Monte Carlo simulations that in exchange coupled FM/AFM multilayers, magnetic dilution leads to a stabilization of domains in the AFM which carry a net magnetization. This magnetization is frozen at low temperatures leading to a frozen exchange field in the FM layer and thus to EB.

These frozen domains depend on the history which means that one has to specify the way the low-temperature state in which the hysteresis loop is calculated (or measured in experiments) is reached. Thus in the diluted case it is necessary to follow exactly the same procedure as is done in experiments: one starts at a temperature well above the Néel temperature with a fully magnetized FM layer (sometimes also with an applied field parallel to this magnetization) and cools the system slowly down to temperatures below the Néel temperature. This cooling process has been done before with Monte Carlo simulations which mimic a dynamical process. However, the dynamical aspect is not important. Rather, the calculation is intended to find local free-energy minima. Therefore, this cooling procedure can alternatively also be done within local mean-field theory in which the local mean field equations which in the present case are given by

$$
m_{i}=\epsilon_{i} \tanh \left[\beta\left(-J_{\mathrm{AF}} \sum_{j} \epsilon_{j} m_{j}+J_{\mathrm{int}} S_{x}+H\right)\right]
$$

are iterated at a fixed temperature until a (metastable) selfconsistent solution is obtained. Then, the temperature is low- 
ered by a small amount and a new iteration process is started with the previously obtained values of the magnetization as initial conditions. This procedure is continued until the final temperature at which the hysteresis curve is going to be calculated is reached. This approach has been applied successfully to random field systems in connection with irreversibilities and frozen states. ${ }^{17}$

It is important to note that the corresponding mean-field energy also fulfills Eq. (5) where on the right-hand side the local magnetization has to be replaced by the local magnetization in the mean-field approximation, i.e.,

$$
\mathcal{F}_{\mathrm{MF}}{ }^{\prime}\left(S_{x}\right)=-2 N l D S_{x}-N l H-J_{\mathrm{int}} \sum_{i \in \text { int }} \epsilon_{i}\left\langle\sigma_{i}\right\rangle .
$$

Cooling the system as described above means that one stays in local minima of the free energy.

The hysteresis loop is calculated in a similar way by changing the external field in small steps solving the meanfield equations numerically in each step by iteration. The AFM interface magnetization $m_{\text {int }}\left(H, S_{x}\right)$ is recorded for each value of $H$ for the descended branch $S_{x}=1$ and the ascended branch $S_{x}=-1$, respectively, and the fields $H_{-}$and $H_{+}$at which the magnetization of the FM switches are then obtained as before, i.e., from $\mathcal{F}^{\prime}=0$ at $S_{x}=1$ and $S_{x}=-1$, respectively, that is, from the implicit equation

$$
H_{ \pm}= \pm 2 D-J_{\mathrm{int}} m_{\mathrm{int}}\left(H_{ \pm}, S_{x}=\mp 1\right) / l .
$$

In these calculations, we use $96 \times 96$ lattice sites per monolayer with periodic boundary conditions in the plane. The fields $H_{ \pm}$depend on the disorder configuration. Therefore, for each degree of dilution, 16 different realizations of the disorder are generated and the fields obtained for each configuration are averaged. The AFM interface magnetization $m_{\text {int }}$ obtained in this way can be decomposed into a sum of two terms, an irreversible (metastable) part $m_{\text {irr }}$ which does not change when going through the hysteresis loop at low temperatures and a part which follows the field, $m_{\mathrm{rev}}$, having two branches for $S_{x}=1$ and $S_{x}=-1$, respectively. Due to this frozen interface magnetization, the fields $H_{+}$and $-H_{-}$ are no longer equal, with the consequence that the system shows exchange bias.

Before we discuss our numerical results, we can go, similar to the undiluted case, one step further noting that in the limit of small effective fields $m_{\text {rev }}$ can be linearized,

$$
m_{\mathrm{rev}}=\chi_{\mathrm{AF}}^{(1)} J_{\mathrm{int}} S_{x}+\chi_{\mathrm{AF}}^{(2)} H .
$$

The first term corresponds to the response to the exchange field while the second term is the response to the applied field. Only for the case of an undiluted AFM monolayer is the induced AFM magnetization strictly proportional to the effective field $J_{\text {int }} S_{x}+H$, in which case both susceptibilities in Eq. (14) are equal.

Thus, within the linear approximation, the AFM interface magnetization which is shifted by $m_{\text {irr }}$ contains two branches during a hysteresis cycle. In the case of $J_{\text {int }}>0$, there is an upper branch for $S_{x}=1$ when reducing the external field and a lower branch after switching of the FM layer from $S_{x}=1$ to $S_{x}=-1$ when increasing the field again. For $J_{\text {int }}<0$, a reversed behavior is observed. This scenario is exactly what is also obtained in our earlier Monte Carlo simulations (see Figs. 4 and 5 in Ref. 8).

Within this linear approximation, explicit equations for the fields $H_{+}, H_{-}$can be obtained from Eq. (13), i.e.,

$$
H_{ \pm}=\frac{ \pm 2 D-J_{\mathrm{int}} m_{\mathrm{irr}} / l \pm J_{\mathrm{int}}^{2} \chi_{\mathrm{AF}}^{(1)} / l}{1+J_{\mathrm{int}} \chi_{\mathrm{AF}}^{(2)} / l},
$$

resulting in the following expressions for the coercive field and the bias field, respectively:

$$
\begin{aligned}
& H_{\mathrm{eb}}=\frac{1}{2}\left(H_{+}+H_{-}\right)=\frac{-J_{\mathrm{int}} m_{\mathrm{irr}} / l}{1+J_{\mathrm{int}} \chi_{\mathrm{AF}}^{(2)} / l}, \\
& H_{c}=\frac{1}{2}\left(H_{+}-H_{-}\right)=\frac{2 D+J_{\mathrm{int}}^{2} \chi_{\mathrm{AF}}^{(1)} / l}{1+J_{\mathrm{int}} \chi_{\mathrm{AF}}^{(2)} / l} .
\end{aligned}
$$

From Eq. (16) it can be concluded that EB only occurs if the interface magnetization contains a part which is frozen during field reversal. Even though this is rather obvious, it is seen here most clearly. Note that the second term in the denominator of Eq. (16) is missing in the usual estimate for the bias field. ${ }^{1}$ This is consistent with our approach since in this estimate a part of an AFM interface magnetization which follows the external field is generally not considered. This linear approximation is expected to be valid for a not too large interface coupling just as in the undiluted case.

These analytic results obtained in the linear approximation contribute to a deeper understanding of bias and coercivity. For a numerical calculation of these quantities, however, it is more convenient to start with the calculation of the interface magnetization $m_{\text {int }}$ during a hysteresis cycle in the way described above. From this quantity, the fields $H_{ \pm}$can be obtained directly using Eq. (13) not invoking the linear approximation. Corresponding results for the bias field and the coercive field for different dilutions of the AFM layer are shown in Fig. 5 for negative interface coupling and in Fig. 6 for positive coupling.

For zero dilution, the coercive fields have a cusp at the onset of the antiferromagnetic order in the AFM layer. For small $L$, this is also the maximum of the coercivity while for $\operatorname{larger} L$ the maximum is slightly shifted to smaller temperatures (cf. Figs. 1 and 2). In the diluted cases the cusp is smeared out. The bias field shows a nearly linear decrease as a function of temperature, in agreement with previous investigations both experimentally and theoretically. It vanishes roughly at that temperature at which the coercive field has its maximum. Note, however, that for stronger dilution there is a pronounced shift of this maximum to temperatures lower than the temperature at which the bias vanishes.

The coercive fields show an interesting behavior at low temperatures where in the diluted case a dip occurs for ferromagnetic interface coupling and an upwards turn for antiferromagnetically coupled FM/AFM layers. This feature has its origin in nearly loose spins in the AFM interface layer which contribute significantly to the susceptibility $\chi_{\mathrm{AF}}^{(2)}$ at low temperatures leading to an enhancement or a depression of the coercive field due to the changing sign of $J_{\text {int }}$ in the denominator in Eq. (17). This sensitivity of the coercive field with respect to the sign of $J_{\text {int }}$ has as an important conse- 


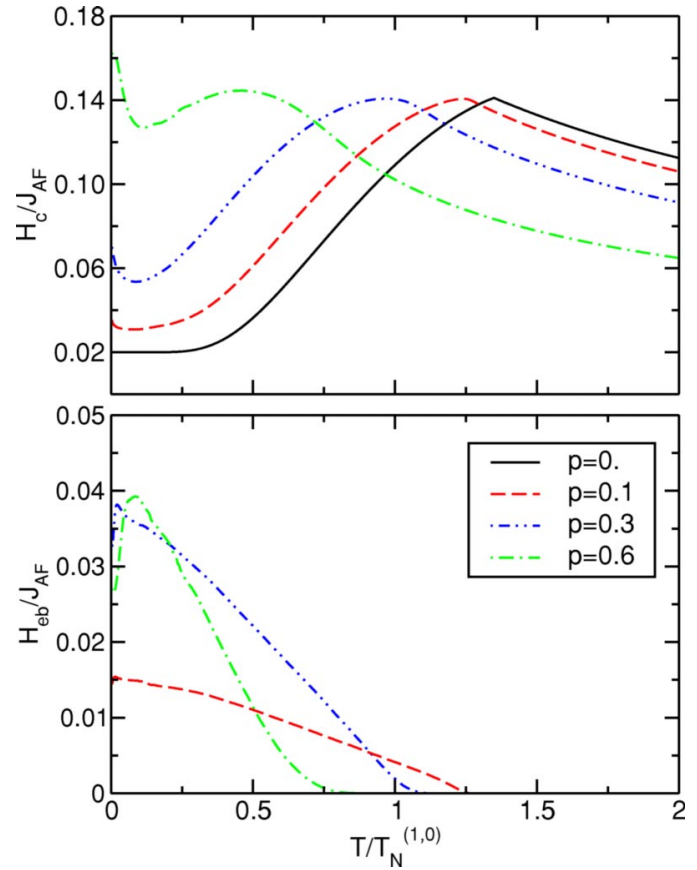

FIG. 5. (Color online) Coercivity and bias field for different dilution of the AFM as a function of reduced temperature. $L=3$ and $J_{\text {int }}=-J_{\mathrm{AF}}$.

quence the possibility of an experimental determination of the sign of the exchange interaction. In Ref. 15, the measured coercive fields of IrMn multilayers show an upwards shift very similar to our findings. Thus it is tempting to conclude that the exchange interaction at the FM/AFM interface in these layers is antiferromagnetic.

\section{CONCLUSION}

In conclusion, we derived in the present paper within a mean-field approach some analytic results for both the coercive field and the bias field from which a deeper insight into the physics of exchange coupled FM/AFM layers can be obtained. For these systems, a significant enhancement of the coercive field was found with a maximum around the Néel temperature. Diluting the AFM layer magnetically in addition to this behavior EB is obtained depending on the degree of dilution in the AFM layer, in complete qualitative agreement with our previous investigations on the domain state model studied with Monte Carlo simulations. In particular,

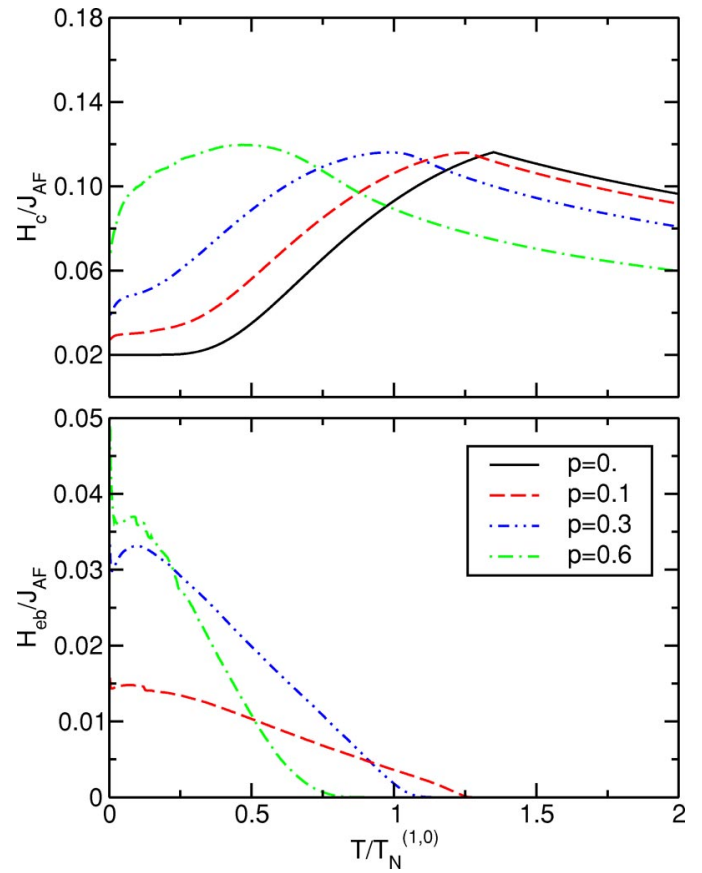

FIG. 6. (Color online) Coercivity and bias field for different dilution of the AFM as a function of reduced temperature. $L=3$ and $J_{\text {Int }}=J_{\mathrm{AF}}$.

the domains formed after field cooling with the mean-field approach have the same structure as those obtained with Monte Carlo simulations. However, there are slight differences as far as the magnitude of the exchange bias fields is concerned, since cooling with the mean-field approach may freeze the system in shallow local minima from which it still could escape within the Monte Carlo approach.

The observed enhancement of the coercive field originates in the coupling of the FM layer to that part of the AFM interface layer magnetization which follows the external field during a hysteresis cycle. Since the AFM layer carries an induced magnetization even above the Néel temperature, the enhancement of the coercivity persists well above this temperature. The bias field, on the other hand, originates in the frozen part of the magnetization of the AFM interface layer. Both are not directly related in the sense that one can change one of them without changing the other one.

\section{ACKNOWLEDGMENTS}

The authors thank B. J. Hickey and C. H. Marrows for valuable discussions. This work was supported by the Deutsche Forschungsgemeinschaft through SFB 491.
*Electronic address: uli@thp.uni-duisburg.de

${ }^{1}$ J. Nogués and I. K. Schuller, J. Magn. Magn. Mater. 192, 203 (1999).

${ }^{2}$ V. Sumryev, S. Stoyanov, J. Zhang, G. Hadjipanayis, D. Givord, and J. Nogués, Nature (London) 423, 850 (2003).

${ }^{3}$ T. C. Schulthess and W. H. Butler, J. Appl. Phys. 85, 5510 (1999).
${ }^{4}$ A. P. Malozemoff, Phys. Rev. B 37, 7673 (1988).

${ }^{5}$ P. Miltényi, M. Gierlings, J. Keller, B. Beschoten, G. Güntherodt, U. Nowak, and K. D. Usadel, Phys. Rev. Lett. 84, 4224 (2000).

${ }^{6}$ M. D. Stiles and R. D. McMichael, Phys. Rev. B 59, 3722 (1999).

${ }^{7}$ D. Suess, M. Kirschner, T. Schrefl, J. Fidler, R. L. Stamps, and J.-V. Kim, Phys. Rev. B 67, 054419 (2003). 
${ }^{8}$ U. Nowak, K. D. Usadel, P. Miltényi, J. Keller, B. Beschoten, and G. Güntherodt, Phys. Rev. B 66, 014430 (2002).

${ }^{9}$ U. Nowak, A. Misra, and K. D. Usadel, J. Magn. Magn. Mater. 240, 243 (2002).

${ }^{10}$ T. Mewes, R. Lopusnik, J. Fassbender, B. Hillebrands, M. Jung, D. Engel, A. Ehresmann, and H. Schmoranzer, Appl. Phys. Lett. 76, 1057 (2000).

${ }^{11}$ A. Mougin, T. Mewes, M. Jung, D. Engel, A. Ehresmann, H. Schmoranzer, J. Fassbender, and B. Hillebrands, Phys. Rev. B 63, 060409 (2001).

${ }^{12}$ H. T. Shi, D. Lederman, and E. E. C. Fullerton, J. Appl. Phys. 91,
7763 (2002).

${ }^{13}$ L. Wee, R. L. Stamps, and R. E. Camley, J. Appl. Phys. 89, 6913 (2001).

${ }^{14}$ B. Beckmann, U. Nowak, and K. D. Usadel, Phys. Rev. Lett. 91, 187201 (2003).

${ }^{15}$ M. Ali, C. H. Marrows, M. Al-Jawad, B. J. Hickey, A. Misra, U. Nowak, and K. D. Usadel, Phys. Rev. B 68, 214420 (2003).

${ }^{16}$ C. Leighton, H. Suhl, M. J. Pechan, R. Compton, J. Nogués, and I. K. Schuller, J. Appl. Phys. 92, 1483 (2002).

${ }^{17}$ G. S. Grest, C. M. Soukoulis, and K. Levin, Phys. Rev. B 33, 7659 (1986). 